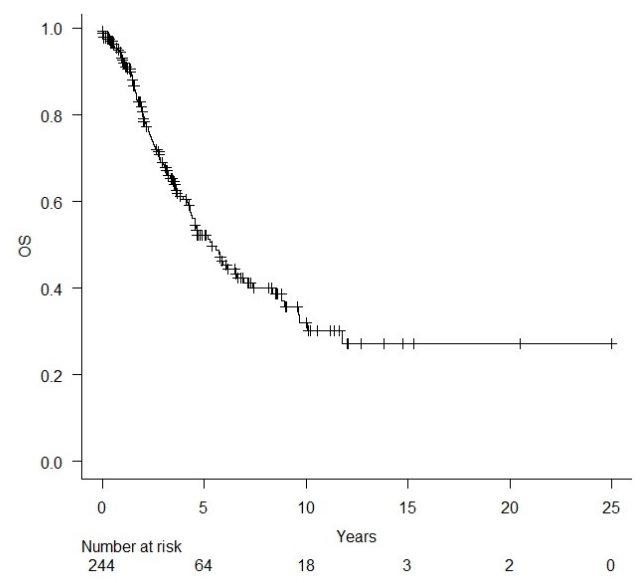

\title{
SURVIVAL OF MYELOMA PATIENTS RECEIVING BISPHOSPHONATES IN A "REAL LIFE" EXPERIENCE: POTENTIAL IMPACT ON RISK OF OSTEONECROSIS OF THE JAW (ONJ)
}

\author{
Federico Monaco ${ }^{1}$, Vittorio Fusco ${ }^{1}$, Giulia Limberti ${ }^{1}$, Antonella Fasciolo ${ }^{1}$, Elisabetta Ferrero ${ }^{1}$, Paola Brigo, Daniela \\ Tartara $^{1}$, Anna Baraldi, Gioacchino Catania ${ }^{1}$, marco ladetto
}

1 Azienda Ospedaliera SS. Antonio e Biagio e Cesare Arrigo

Funding: The author(s) received no specific funding for this work.

Potential competing interests: The author(s) declared that no potential competing interests exist.

\section{Abstract}

Background. Expected survival of myeloma patients increased in recent decades, after introduction of new drugs and strategies. Median survival has been reported about 5 years, but it is very variable, according to age, comorbidities, disease stage, etc.

Almost all myeloma patients receive bisphosphonates as antiresorptive treatment (with denosumab recently approved as an alternative) shortly after diagnosis, and 1\%-15\% of them develop Osteonecrosis of the Jaw (ONJ), within some years of treatment (or observation). Expected survival of myeloma patients can influence the choice of ONJ treatment among possible options.

Methods. Charts of myeloma patients receiving bisphosphonates and observed at the Haematology Unit of Alessandria Hospital in years 2005-2020 have been reviewed. Kaplan-Meier actuarial survival was evaluated.

Results. Median survival from the start of antiresorptive treatment of 244 patients was 5.36 years - with $79 \%, 69 \%, 60 \%$, $52 \%$ of patients alive at 2, 3, 4, 5 years. At 7 and 10 years, $41.3 \%$ and $30.2 \%$ of patients were alive. 
ONJ occurred in 7 patients, after a median of 6.3 years (range 0.5-13.2) from the start of treatment. Mean survival from the ONJ diagnosis time of those 7 patients was 2.9 years (range $0.5-6.0$ ).

Conclusions: Expected survival of myeloma patients in a "real life" setting after the start of antiresorptive treatment is medially long in terms of years, with a large portion of patients exposed at risk of developing ONJ for more than 5 years. A surgical treatment of $\mathrm{ONJ}$ is warranted, being expected survival not so short to hamper jawbone surgery.

Background: Despite significant advances in treatment and improvements in overall survival ${ }^{[1][2][3][4]}$, multiple myeloma has reported as incurable ${ }^{[5]}$. The median survival is just over 5 years, and most patients receive four or more different lines of therapy throughout their disease course. Almost all myeloma patients receive bisphosphonates (or - only recently denosumab) to reduce the risk of Skeletal Related Events (SRE) and are at risk of Osteonecrosis of the Jaw (ONJ). As survival prolongs, a large proportion of myeloma patients is at risk for ONJ for several years. The length of survival from the start of antiresorptive treatment and the length of survival after ONJ diagnosis are both of potential impact when choosing the ONJ treatment (surgical versus not surgical), but few data have been published on this issue.

Materials and Methods: We reviewed all myeloma patients affected by bone lesions observed by our multidisciplinary team and treated at the Hematology Unit in years 2005-2020. We examined birth date, date of start of antiresorptive treatment, type of drug (pamidronate; zoledronic acid; other drugs or sequences), date of diagnosis of ONJ, date of death (or latest follow-up visit). We obtained actuarial survival curves by Kaplan-Meier method.

Results: We evaluated survival data of 244 patients. Median survival from the start of antiresorptive treatment (pamidronate or zoledronic acid) was 5.36 years - with $79 \%, 69 \%, 60 \%, 52 \%$ of patients alive at 2, 3, 4, 5 years. At 7 and 10 years, $41.3 \%$ and $30.2 \%$ of patients were alive. (Figure 1 ).

ONJ occurred in 7 patients, after a median of 6.3 years (range 0.5-13.2) from the start of treatment. Survival of those 7 patients ranged between 2 and 14.5 years (median 10.5 years). Mean survival after the ONJ diagnosis was 2.9 years (range 0.5-6.0) in the 7 patients. 


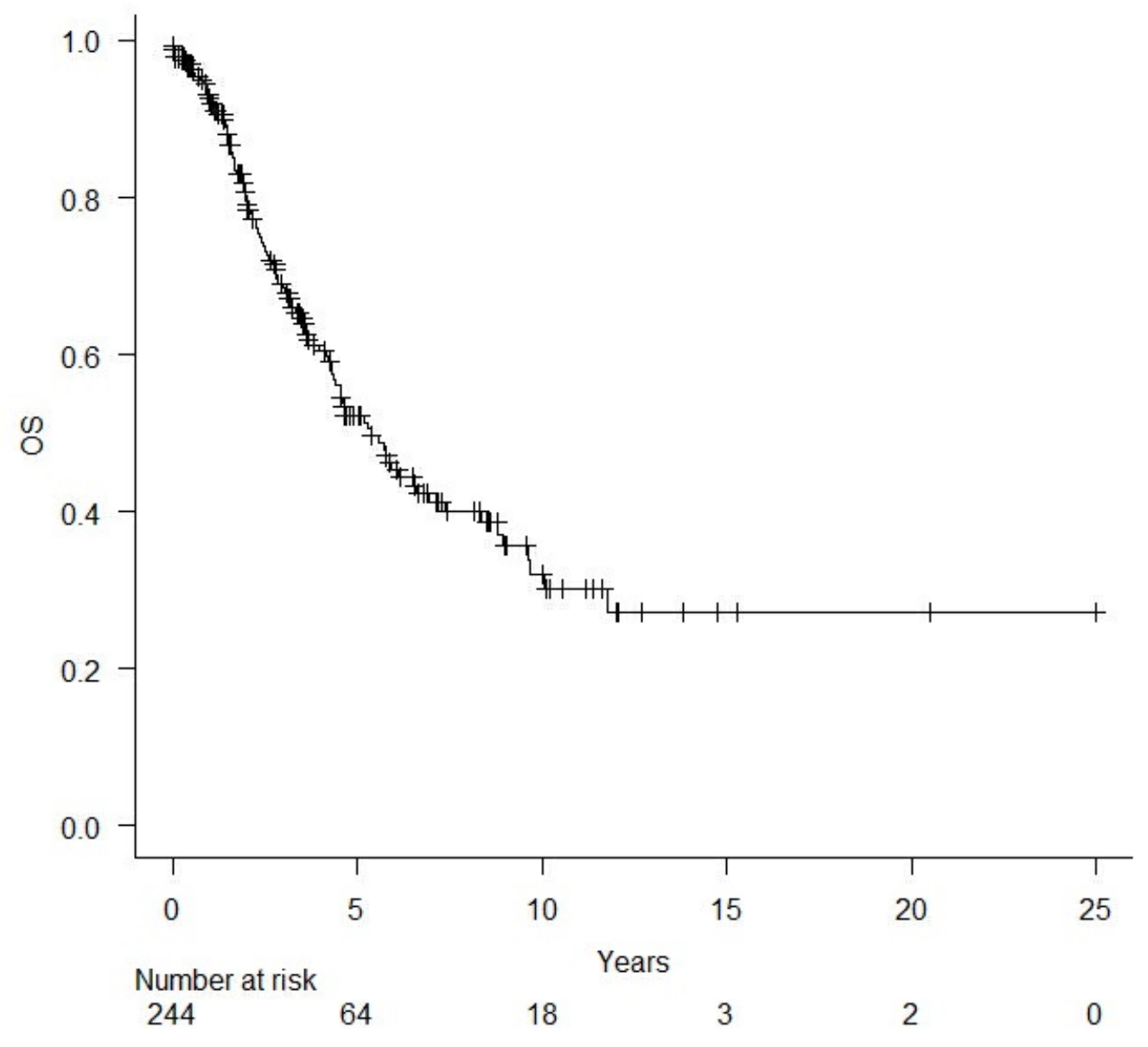

Figure 1. Actuarial survival of Myeloma patients (Kaplan Meier)

Conclusions: Expected survival of myeloma patients in a "real life" setting after the start of antiresorptive treatment is medially long (more than five years), with a large portion of patients exposed for several years to risk of ONJ. The comparison of this survival with that of large populations of patients developing ONJ[6][7] might be of value: a) to predict the ONJ risk among general population of myeloma patients; b) to establish the real ONJ risk in patients surviving more than one or two years (and to choose the best antiresorptive treatment duration and schedule); c) to evaluate feasibility and opportuneness of surgical treatment of ONJ.

\section{References}

1. `Dianne Pulte, Lina Jansen, Hermann Brenner. (2020). Changes in long term survival after diagnosis with common hematologic malignancies in the early 21st century. Blood Cancer J., vol. 10 (5). doi:10.1038/s41408-020-0323-4.

2. 'Samantha Pozzi, Luigi Marcheselli, Alessia Bari, Eliana V. Liardo, et al. (2013). Survival of multiple myeloma patients in the era of novel therapies confirms the improvement in patients younger than 75 years: a population-based analysis. Br J Haematol, vol. 163 (1), 40-46. doi:10.1111/bjh.12465.

3. ^Dianne Pulte, Lina Jansen, Felipe A. Castro, Katharina Emrich, et al. (2015). Trends in survival of multiple myeloma 
patients in Germany and the United States in the first decade of the 21st century. Br J Haematol, vol. 171 (2), 189-196. doi:10.1111/bjh.13537.

4. 'Philippe Moreau, Michel Attal, Thierry Facon. (2015). Frontline therapy of multiple myeloma. doi:10.1182/blood-201409-568915.

5. `Joseph Mikhael, Nofisat Ismaila, Matthew C. Cheung, Caitlin Costello, et al. (2019). Treatment of Multiple Myeloma: ASCO and CCO Joint Clinical Practice Guideline. JCO, vol. 37 (14), 1228-1263. doi:10.1200/jco.18.02096.

6. 'Vittorio Fusco, MARCO CABRAS, Alessio Gambino, Massimo DI MAIO, et al. (2021). Expected survival of cancer patients and myeloma undergoing bisphosphonates or denosumab. Potential impact on diagnosis and management of Osteonecrosis of Jaws (ONJ). Qeios. doi:10.32388/MQ2/YG.

7. 'MARCO CABRAS, Alessio Gambino, DAVIDE CONROTTO, Marco Garrone, et al. (2021). Survival of metastatic cancer patients and myeloma after diagnosis of Osteonecrosis of Jaws (ONJ). Potential impact on ONJ treatment strategies.. Qeios. doi:10.32388/IKLIWH. 